\title{
Differences in plasma and sputum biomarkers between COPD and COPD-asthma overlap
}

\author{
Hiroshi Iwamoto ${ }^{1,2,6}$, Jing Gao ${ }^{1,6}$, Jukka Koskela ${ }^{3}$, Vuokko Kinnula ${ }^{1, \dagger}$, \\ Hideo Kobayashi ${ }^{4}$, Tarja Laitinen ${ }^{5}$ and Witold Mazur ${ }^{1}$
}

Affiliations: 'Dept of Medicine, Pulmonary Division, University of Helsinki and Helsinki University Central Hospital, Helsinki, ${ }^{3}$ Dept of Medicine, University of Helsinki, Helsinki, and ${ }^{5}$ Dept of Pulmonary Diseases, Turku University Central Hospital, Turku, Finland. ${ }^{2}$ Dept of Molecular and Internal Medicine, Graduate School of Biomedical Sciences, Hiroshima University, Hiroshima, and ${ }^{4}$ Dept of Pulmonary Medicine, National Defense Medical College, Tokorozawa, Japan. ${ }^{6}$ These authors contributed equally to this work.

Correspondence: W. Mazur, Dept of Medicine, Pulmonary Division, University of Helsinki and Helsinki University Central Hospital, Haartmaninkatu 4, 00014 Helsinki, Finland. E-mail: iwamotohiroshigagmail.com

ABSTRACT The pathophysiological features of chronic obstructive pulmonary disease (COPD)-asthma overlap are poorly understood and there has been no study of plasma or sputum biomarkers in overlap patients. In order to clarify the similarity and differences between overlap and COPD or asthma, we have investigated four potential biomarkers of COPD: surfactant protein A (SP-A), soluble receptor for advanced glycation end-products (sRAGE), myeloperoxidase (MPO) and neutrophil gelatinase-associated lipocalin (NGAL). SP-A and sRAGE are pneumocyte-derived markers. MPO and NGAL are neutrophil-derived molecules, but NGAL can also be expressed by respiratory epithelial cells.

Plasma levels of SP-A and sRAGE and induced sputum levels of MPO and NGAL were measured by enzyme immunoassay/ELISA in 134 subjects: nonsmokers $(n=26)$, smokers $(n=23)$, asthma $(n=32)$, COPD $(n=39)$ and COPD-asthma overlap patients $(n=14)$.

In patients with COPD-asthma overlap, sputum MPO and plasma SP-A were significantly elevated whereas plasma sRAGE levels were reduced compared with asthma patients. Only sputum NGAL was significantly elevated in COPD-asthma overlap compared with COPD $(p=0.00016)$ and could be used to differentiate patients with overlap from those with COPD.

Increased induced sputum levels of NGAL might be a characteristic feature of overlap, suggesting enhanced neutrophilic airway inflammation and/or airway epithelial injury in COPD-asthma overlap.

@ERSpublications

Increased sputum levels of neutrophil gelatinase-associated lipocalin in COPD-asthma overlap versus COPD/asthma patients http://ow.ly/qxC8X

Support statement: This work was financially supported by the EVO funding of the Helsinki University Central Hospital, University of Helsinki, Finnish Antituberculosis Association Foundation, and partly by the SalWe Research programme for IMO (Tekes - the Finnish Funding Agency for Technology and Innovation grant number 648/10).

Conflict of interest: Disclosures can be found alongside the online version of this article at www.erj.ersjournals.com 


\section{Introduction}

Together, asthma and chronic obstructive pulmonary disease (COPD) are the most common chronic lung diseases worldwide. Differential diagnosis of asthma and COPD is important because of the different therapeutic approaches and the distinct clinical outcomes in morbidity and mortality $[1,2]$. Although asthma and COPD differ from each other in their patterns of inflammation, their immunological mechanisms and the extent of the reversibility of airflow limitation, a significant number of patients exhibit symptoms and signs that are associated with both conditions [1-3]. The coexistence of asthma and COPD can frequently be seen in the general population, especially in elderly patients [4]. Importantly, these overlap patients might have distinct clinical characteristics, such as lower health-related quality of life or increased frequency of exacerbation when compared with patients with COPD alone with the same degree of lung function abnormality $[5,6]$.

Spirometry is essential in the diagnosis and assessment of severity of COPD, but it has limited efficacy in the evaluation of symptom burden and in helping to make a differential diagnosis from asthma. Furthermore, there is an urgent need to clarify the clinically relevant COPD phenotype as well as the underlying biological mechanisms [7]. In order to elucidate the pathophysiology of COPD-asthma overlap, we have devised a panel of four potential biomarkers. Two of the biomarkers studied here are previously well-characterised neutrophil-derived inflammatory molecules, myeloperoxidase (MPO) and neutrophil gelatinase-associated lipocalin (NGAL), both of which are associated with inflammation and early airway injury in smokingrelated diseases [8]. Recently using two-dimensional electrophoresis and mass spectrometry we have shown that surfactant protein A (SP-A), which has multiple immunomodulatory roles in lung host defence, is associated with cigarette smoke-induced lung injury and in the development of COPD [9-11]. The receptor for advanced glycation end-products (RAGE) is a multi-ligand receptor abundantly expressed in type I pneumocytes. The expression of RAGE is significantly decreased in COPD lung, especially in severe disease [12]. The soluble isoform of RAGE (sRAGE) in plasma might represent a potential marker of COPD, as its levels decline in patients with COPD and decrease further during exacerbations [13-15].

We hypothesised that patients with asthma, COPD and COPD-asthma overlap would differ in their biomarker profiles. The aim of the present study was to determine the plasma and induced sputum levels of the four potential biomarkers of COPD in patients with asthma, COPD and COPD-asthma overlap. Another goal was to investigate whether the levels of these markers would be associated with variables such as pack-years, lung function and sputum cell profiles.

\section{Methods}

Subjects

Plasma and induced sputum samples were collected from 134 volunteer individuals. Based on their medical history and self-reported questionnaire data, the study subjects were categorised into five groups: healthy nonsmokers (NS, $\mathrm{n}=26$ ) and asymptomatic healthy smokers with normal lung function (HS, $\mathrm{n}=23$ ), patients with asthma (asthma, $n=32$ ), patients with COPD (COPD, $n=39$ ), and patients with asthma and irreversible obstruction (COPD-asthma overlap, $n=14$ ). The patients, who are part of the longitudinally followed cohort of Finnish asthma and COPD patients (FinnCADStudy) [5, 16], were recruited consecutively from Helsinki University Central Hospital (HUCH). The control subjects consisted of responders to an advertisement in HUCH and the local media. Spirometry and the diffusing capacity of the lung for carbon monoxide ( $D$ LCO) were performed on all participants. Spirometric values were assessed by standard spirometry (Medikro M 903; Medikro Oy, Kuopio, Finland) and were performed according to American Thoracic Society (ATS)/European Respiratory Society (ERS) recommendations [17]. Reference values were obtained from Finnish reference values for spirometry [18].

The diagnosis of asthma was based on the British Guidelines on Asthma Management [19], with one or more of the following findings together with the clinical history compatible of asthma: a postbronchodilator increase in forced expiratory volume in $1 \mathrm{~s}(\mathrm{FEV} 1)$ of $\geqslant 12 \%$, a bronchodilator response of $\geqslant 15 \%$ or diurnal variation of $\geqslant 20 \%$ in peak expiratory flow (PEF) recording, moderate-to-severe bronchial hyperreactivity, or a decrease in $\mathrm{FEV} 1$ of $\geqslant 15 \%$ in the exercise test. COPD was diagnosed according to the ATS/ERS Task Force recommendations $[16,20]$ and defined by irreversible chronic airway obstruction (FEV1/forced vital capacity (FVC) $<0.70$ or $\mathrm{FEV} 1 / \mathrm{FVC}<88 \%$ predicted) in postbronchodilation spirometry related to long-term smoking $[1,18]$. The diagnosis of COPD-asthma overlap was made when patients fulfilled both diagnostic criteria for asthma and COPD. All the cases with COPD and overlap had no history of $\alpha_{1}$-antitrypsin deficiency in their family. No patients were either receiving oral steroid treatment or experienced an exacerbation during the month before inclusion. Control subjects were neversmokers or current smokers with normal lung function and no history of lung disease. 
This study was approved by the Ethics Committees Helsinki University Central Hospital and conducted in accordance with the ethical standards established in the Helsinki Declaration of 1975. All participants provided written informed consent.

\section{Collection of blood and induction of sputum samples}

Peripheral whole venous blood was collected into EDTA tubes, plasma was prepared by centrifugation for $10-15 \mathrm{~min}$ at $4500 \mathrm{rpm}$ and stored at $-80^{\circ} \mathrm{C}$ until analysed. Sputum was induced by inhalation of hypertonic saline and treated with dithioerythritol (Sigma, Munich, Germany) as recommended by the ERS Task Force and described in detail previously $[21,22]$. The supernatant was frozen at $-80^{\circ} \mathrm{C}$ for biochemical analyses. Cell viability was studied with trypan blue in a Burker chamber [23]. Cytocentrifuge preparations were made by Cytospin (Thermo Scientific, Wilmington, DE, USA) and centrifuged at $4053 \times g$ for 6 min. The slides were stained by May-Grunwald-Giemsa staining (Merck, Darmstadt, Germany) for cell differential counts with 400 cells being counted from each slide. Detailed cell profiles were assessed for all sputum samples. Only the samples with $<70 \%$ of squamous epithelial cells were accepted for further assessments. The slides were frozen at $-20^{\circ} \mathrm{C}[23]$.

\section{Measurement of SP-A, sRAGE, MPO and NGAL in plasma and sputum supernatants}

SP-A, sRAGE, MPO and NGAL levels were measured by commercially available enzyme immunoassay/ ELISA kits (Kokusai-F kit (Sysmex, Kobe, Japan), R\&D Systems (Minneapolis, MN, USA), Abnova Inc. (Walnut, CA, USA), and Uscn Life Science Inc. (Wuhan, China), respectively) according to the manufacturers' instructions. The detection limits of sRAGE, SP-A, MPO and NGAL were $78 \mathrm{pg} \cdot \mathrm{mL}^{-1}$, $1 \mathrm{ng} \cdot \mathrm{mL}^{-1}, 0.78 \mathrm{ng} \cdot \mathrm{mL}^{-1}$ and $0.039 \mathrm{ng} \cdot \mathrm{mL}^{-1}$, respectively.

\section{Statistical analysis}

The results are expressed as the mean \pm SEM. Comparisons between groups were evaluated using repeated measures ANOVA followed by Fisher's protected least significant difference test (PLSD) post hoc comparison. Plasma and sputum biomarkers were further analysed by plotting receiver operating characteristic (ROC) curves for their predictive capability in distinguishing patients with overlap from those with COPD, and patients with overlap and COPD from those with asthma or NS. Correlations of the plasma and sputum markers with demographics, lung function and sputum cell profiles were calculated by Spearman's rank correlations. To obtain an independent predictor for each biomarker, multivariate stepwise regression analysis was performed. A p-value of $<0.05$ was considered statistically significant. All statistical analyses were performed with the SPSS 16.0 software program (SPSS Inc., Chicago, IL, USA).

\section{Results}

\section{Subject characteristics}

The demographics and clinical characteristics of the subjects are shown in table 1. The patients were older than the control subjects. Patients with COPD and overlap had more pack-years and significantly lower FEV1 and DLCO than those in the NS, HS and asthma groups (table 1) (ANOVA followed by post hoc Fisher's PLSD; data not shown). The overlap patients had a greater improvement in the reversibility test and diurnal variation of PEF compared with asthma or COPD patients. A confirmation of the presence of asthma was usually made by an improvement in FEV1 after a bronchodilator, in 14 cases by measurements of diurnal variation of PEF and in six by an increase in bronchial responsiveness. None of the participants had experienced an exacerbation or a respiratory tract infection in the month preceding the study.

Sputum was induced from all the subjects, but, based on the evaluation of cell profiles, 99 samples were accepted for further analysis: NS $(n=18)$, HS $(n=17)$, asthma $(n=25)$, COPD $(n=28)$ and overlap $(n=11)$. The patients with asthma and overlap had a higher percentage of sputum eosinophils when compared with NS, HS and COPD groups (table 1). The percentage of sputum neutrophils was higher in COPD and overlap compared with NS and HS.

Plasma sRAGE decreases and plasma SP-A increases in COPD and COPD-asthma overlap The plasma levels of sRAGE were significantly decreased in COPD and overlap groups $(715.8 \pm 51.2$ and $790.6 \pm 81.8 \mathrm{pg} \cdot \mathrm{mL}^{-1}$, respectively) when compared with asthma groups $(\mathrm{p}=0.025$ and $\mathrm{p}=0.013$, respectively) (fig. 1a). The plasma levels of SP-A were elevated in the COPD and overlap groups $\left(45.5 \pm 4.1\right.$ and $54.5 \pm 7.5 \mathrm{ng} \cdot \mathrm{mL}^{-1}$, respectively) compared with the NS, HS and asthma groups (fig. 1b). There was no significant difference in plasma SP-A levels between the COPD and overlap groups $(\mathrm{p}=0.124)$. 
TABLE 1 Characteristics of the subjects

\begin{tabular}{|c|c|c|c|c|c|}
\hline & NS & HS & Asthma & COPD & Overlap \\
\hline Subjects $n$ & 26 & 23 & 32 & 39 & 14 \\
\hline Females/males $\mathrm{n}$ & $10 / 16$ & $11 / 12$ & $16 / 16$ & $13 / 26$ & $5 / 9$ \\
\hline Age years & $46.9 \pm 2.6$ & $47.6 \pm 2.5$ & $58.3 \pm 1.5$ & $62.4 \pm 1.1$ & $61.2 \pm 1.5$ \\
\hline BMI $\mathrm{kg} \cdot \mathrm{m}^{-2}$ & $28.2 \pm 1.0$ & $26.9 \pm 1.0$ & $27.5 \pm 1.0$ & $25.5 \pm 0.7$ & $28.3 \pm 1.9$ \\
\hline \multicolumn{6}{|l|}{ Smoking status } \\
\hline Former smoker & 0 & 0 & $17(53)$ & $13(33)$ & 8 (57) \\
\hline Current smoker & 0 & $23(100)$ & $4(13)$ & $26(67)$ & $6(43)$ \\
\hline Pack-years & 0 & $25.8 \pm 3.7$ & $16.1 \pm 3.8$ & $47.8 \pm 3.8$ & $36.9 \pm 6.1$ \\
\hline Allergy & $4(15)$ & $9(\overline{40})$ & $18(56)$ & $11(28)$ & $5(\overline{36})$ \\
\hline ICS use & 0 & 0 & $28(88)$ & $13(33)$ & $14(100)$ \\
\hline \multicolumn{6}{|l|}{ Postbronchodilator } \\
\hline FVC L & $4.7 \pm 0.2$ & $4.4 \pm 0.3$ & $3.5 \pm 0.2$ & $3.2 \pm 0.2$ & $3.2 \pm 0.3$ \\
\hline FEV1 L & $3.9 \pm 0.2$ & $3.5 \pm 0.2$ & $2.6 \pm 0.1$ & $1.9 \pm 0.1$ & $1.9 \pm 0.2$ \\
\hline FEV $1 \%$ pred & $103.8 \pm 2.1$ & $96.0 \pm 3.6$ & $77.0 \pm 3.0$ & $57.9 \pm 3.2$ & $55.3 \pm 3.1$ \\
\hline $\mathrm{FEV}_{1} / \mathrm{FVC}$ & $83.9 \pm 1.1$ & $80.1 \pm 1.3$ & $80.9 \pm 1.7$ & $62.6 \pm 2.4$ & $69.9 \pm 4.1$ \\
\hline DLCo $\%$ pred & $93.8 \pm 4.6$ & $89.4 \pm 2.8$ & $87.8 \pm 5.6$ & $62.9 \pm 4.2$ & $66.8 \pm 5.2$ \\
\hline$\Delta \mathrm{FEV}{ }_{1}^{\#} \mathrm{~mL}$ & $110 \pm 31$ & $78 \pm 38$ & $173 \pm 36$ & $109 \pm 30$ & $203 \pm 49$ \\
\hline$\Delta \mathrm{FEV} 11^{\#} \%$ & $3.1 \pm 0.9$ & $2.4 \pm 1.1$ & $7.8 \pm 1.6$ & $7.5 \pm 1.3$ & $13.0 \pm 4.0$ \\
\hline PEF diurnal variability \% & & & $28.1 \pm 2.0$ & $17.2 \pm 1.8$ & $37.9 \pm 4.8$ \\
\hline \multicolumn{6}{|c|}{ Differential sputum cell counts \% } \\
\hline Eosinophils & $1.5 \pm 0.8$ & $0.9 \pm 0.6$ & $6.3 \pm 1.9$ & $1.4 \pm 0.5$ & $8.3 \pm 3.5$ \\
\hline Neutrophils & $34.7 \pm 2.7$ & $41.9 \pm 5.4$ & $53.4 \pm 4.6$ & $63.1 \pm 3.6$ & $68.2 \pm 4.1$ \\
\hline Macrophages & $59.11 \pm 3.3$ & $54.3 \pm 5.6$ & $41.3 \pm 4.4$ & $32.9 \pm 3.7$ & $21.8 \pm 3.5$ \\
\hline
\end{tabular}

Data are presented as mean \pm SEM or $n(\%)$, unless otherwise stated. NS: nonsmokers; HS: healthy smokers; COPD: chronic obstructive pulmonary disease; BMI: body mass index; ICS: inhaled corticosteroid; FVC: forced vital capacity; FEV1: forced expiratory volume in $1 \mathrm{~s} ; \%$ pred: \% predicted; DLCO: diffusing capacity of the lung for carbon monoxide; $\Delta$ : change in; PEF: peak expiratory flow. " : improvement in FEV1 after $400 \mu \mathrm{g}$ salbutamol.

Sputum NGAL increases in COPD-asthma overlap compared with COPD

The sputum levels of MPO were elevated in the overlap group $\left(22.3 \pm 5.1 \mu \mathrm{g} \cdot \mathrm{mL}^{-1}\right)$ when compared with the NS, HS and asthma groups (fig. 2a). Sputum MPO levels were also significantly elevated in COPD compared with asthma. There was no significant difference in sputum MPO levels between COPD and
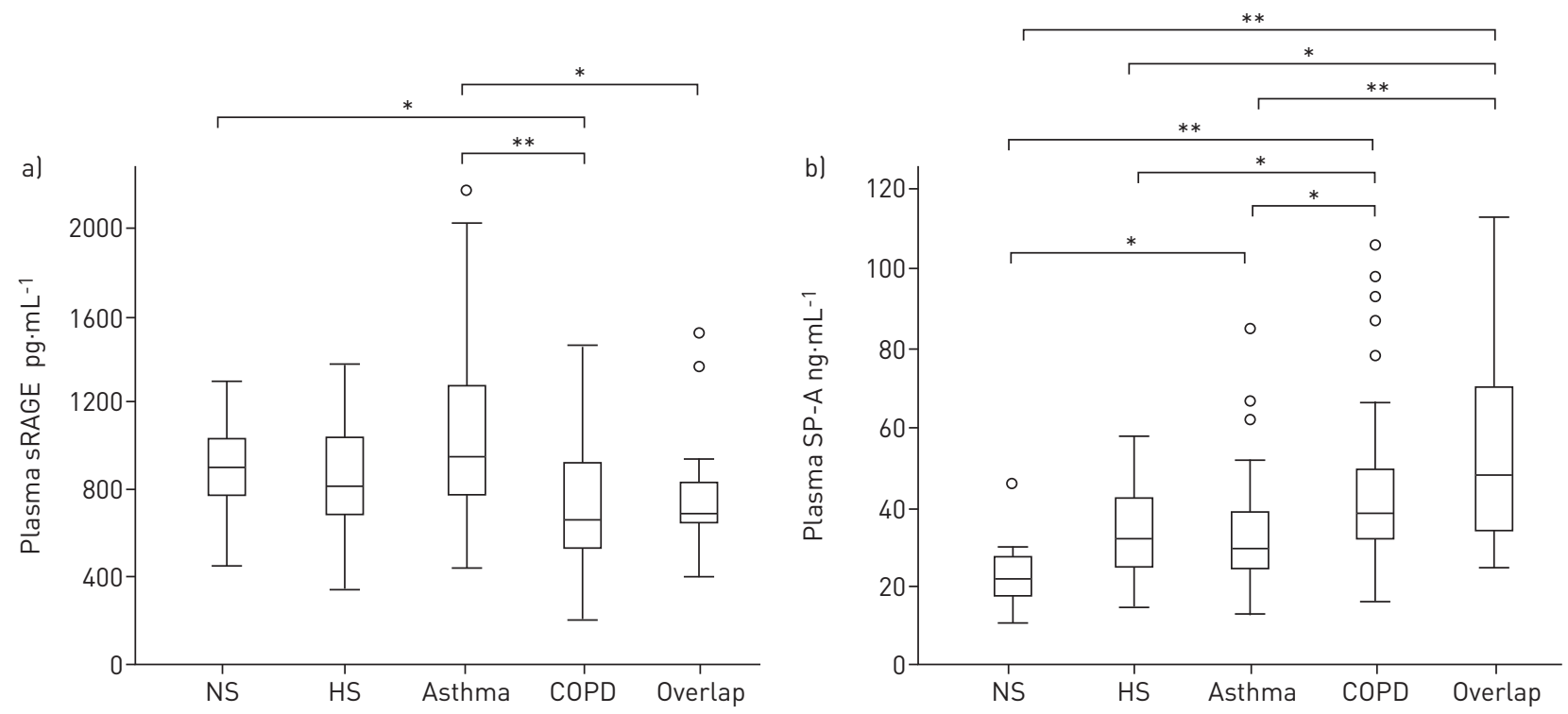

FIGURE 1 Plasma levels of a) soluble receptor for advanced glycation end-products (sRAGE) and b) surfactant protein A (SP-A). The boxes represent the 25th to 75th percentiles, the solid lines within the boxes show the median values, the whiskers are the 10th and 90th centiles, and the circles represent outliers. p-values were calculated by ANOVA followed by Fisher's protected least significant difference test. NS: nonsmoker; HS: healthy smoker; COPD: chronic obstructive pulmonary disease. ${ }^{*}: \mathrm{p}<0.05 ;{ }^{* *}: \mathrm{p}<0.01$. 

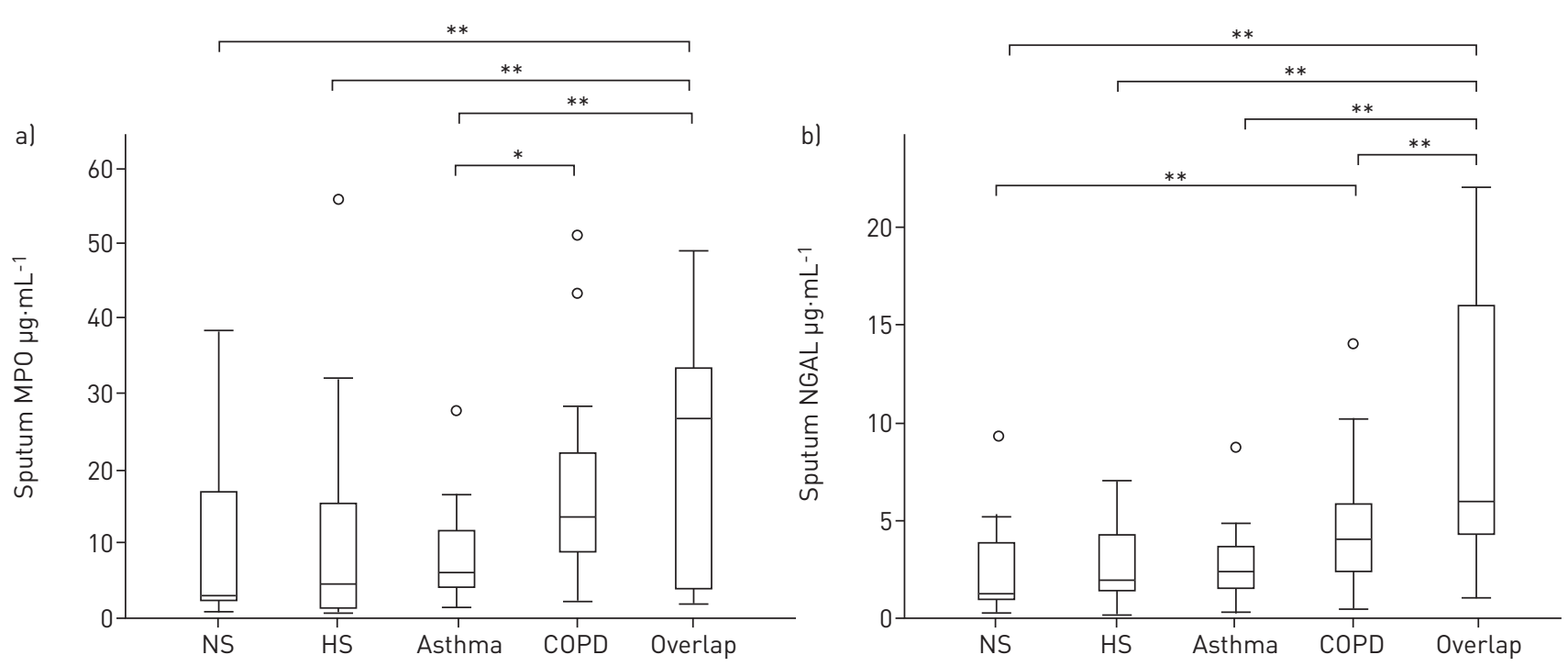

FIGURE 2 Sputum levels of a) myeloperoxidase (MPO) and b) neutrophil gelatinase-associated lipocalin (NGAL). The boxes represent the 25th to 75th percentiles, the solid lines within the boxes show the median values, the whiskers are the 10th and 90th percentiles, and the circles represent outliers. p-values were calculated by ANOVA followed by Fisher's protected least significant difference test. NS: nonsmoker; HS: healthy smoker; COPD: chronic obstructive pulmonary disease. ${ }^{*}: \mathrm{p}<0.05 ;{ }^{*}: \mathrm{p}<0.01$.

overlap $(\mathrm{p}=0.129)$. In contrast, sputum levels of NGAL in overlap $\left(9.1 \pm 2.2 \mu \mathrm{g} \cdot \mathrm{mL}^{-1}\right)$ were significantly higher than in COPD ( $\mathrm{p}=0.00016$ ) (fig. 2b). There was also a significant difference in the sputum levels of NGAL between overlap and NS, HS and asthma, and NS and COPD. Sputum NGAL values did not differ significantly between COPD and asthma, although there was an increasing trend in COPD $(\mathrm{p}=0.058)$.

\section{Sputum NGAL can differentiate COPD-asthma overlap from COPD}

An ROC curve analysis was carried out to evaluate the sensitivity, specificity and diagnostic accuracy of the biomarkers (table 2). The area under the curve (AUC) statistic showed that all of the four markers could differentiate COPD and overlap from nonsmokers (ROC AUC >0.7). All these markers were also useful in distinguishing overlap from asthma patients, and plasma sRAGE and sputum MPO could distinguish patients with COPD from those with asthma. However, only sputum NGAL could distinguish patients with overlap from those with COPD.

\section{Sputum NGAL is significantly associated with airflow obstruction independent of sputum neutrophil level}

The study subjects were pooled in order to analyse the correlations between biomarker concentrations and different variables. Plasma sRAGE levels correlated with DLCO in both univariate and multivariate analyses (tables 3 and 4). Plasma SP-A levels correlated with pack-years with the highest correlation coefficients found in the univariate analysis. However, multivariate analysis revealed FEV1/FVC to be the independent predictor for plasma SP-A. Interestingly, while sputum MPO levels were independently correlated with sputum neutrophil levels, sputum NGAL levels were associated with \% pred FEV1, independently of sputum neutrophil (table 4).

\section{Discussion}

The present study evaluated plasma and sputum levels of four inflammatory and lung injury-related biomarkers in patients with COPD-asthma overlap, COPD alone and asthma alone, and in control subjects. Patients with overlap and COPD displayed a similar pattern of abnormalities in plasma SP-A and sRAGE and sputum MPO. Plasma SP-A and sputum MPO levels were elevated, while plasma sRAGE levels were decreased in the patients with overlap and COPD compared with asthma patients. Overall, AUC statistics revealed that these three markers could differentiate COPD-asthma overlap and COPD from asthma and nonsmoking controls, but they did not distinguish between overlap and COPD. In contrast, only sputum levels of NGAL were significantly elevated in patients with overlap when compared with COPD, and sputum NGAL could differentiate patients with overlap from COPD patients, as well as from asthma and NS. Therefore, elevated levels of sputum NGAL might be a characteristic feature of COPD-asthma overlap. 
TABLE 2 Receiver-operating characteristic (ROC) analysis for plasma and sputum biomarkers

\begin{tabular}{|c|c|c|c|c|c|}
\hline & $\begin{array}{l}\text { Overlap versus } \\
\text { COPD }\end{array}$ & $\begin{array}{l}\text { Overlap versus } \\
\text { asthma }\end{array}$ & $\begin{array}{l}\text { COPD versus } \\
\text { asthma }\end{array}$ & $\begin{array}{c}\text { Overlap versus } \\
\text { NS }\end{array}$ & $\begin{array}{c}\text { COPD versus } \\
\text { NS }\end{array}$ \\
\hline \multicolumn{6}{|l|}{ Plasma } \\
\hline \multicolumn{6}{|l|}{ sRAGE } \\
\hline $\operatorname{AUC}(95 \% \mathrm{CI})$ & $0.570(0.397-0.743)$ & $0.719(0.553-0.884)$ & $0.737(0.616-0.858)$ & $0.703(0.513-0.893)$ & $0.703(0.564-0.842)$ \\
\hline Optimal threshold $\mathrm{pg} \cdot \mathrm{mL}^{-1}$ & $>673.9$ & $<758.2$ & $<903.5$ & $<768.9$ & $<819.7$ \\
\hline Sensitivity/specificity & $0.643 / 0.531$ & $0.714 / 0.812$ & $0.719 / 0.656$ & $0.714 / 0.773$ & $0.625 / 0.727$ \\
\hline $\operatorname{A\cup C}(95 \% \mathrm{CI})$ & $0.607(0.424-0.790)$ & $0.759(0.608-0.910)$ & $0.682(0.550-0.813)$ & $0.935(0.857-1.013)$ & $0.897(0.810-0.985)$ \\
\hline Optimal threshold $\mathrm{ng} \cdot \mathrm{mL}^{-1}$ & $>39.5$ & $>39.0$ & $>33.5$ & $>29.0$ & $>28.5$ \\
\hline Sensitivity/specificity & $0.643 / 0.562$ & $0.643 / 0.750$ & $0.656 / 0.625$ & $0.857 / 0.913$ & $0.844 / 0.913$ \\
\hline $\mathrm{p}$-value & 0.252 & 0.006 & 0.013 & 0.00001 & 0.000001 \\
\hline \multicolumn{6}{|l|}{ Sputum } \\
\hline \multicolumn{6}{|l|}{ MPO } \\
\hline \multicolumn{6}{|l|}{ NGAL } \\
\hline $\operatorname{AUC}(95 \% \mathrm{CI})$ & $0.708(0.502-0.914)$ & $0.818(0.635-1.001)$ & $0.697(0.555-0.839)$ & $0.869(0.728-1.009)$ & $0.781(0.634-0.928)$ \\
\hline Optimal threshold $\mu \mathrm{g} \cdot \mathrm{mL}^{-1}$ & $>4.4$ & $>4.2$ & $>3.5$ & $>4.2$ & $>2.0$ \\
\hline Sensitivity/specificity & $0.727 / 0.714$ & $0.818 / 0.800$ & $0.643 / 0.720$ & $0.818 / 0.889$ & $0.857 / 0.667$ \\
\hline$p$-value & 0.046 & 0.003 & 0.014 & 0.001 & 0.001 \\
\hline
\end{tabular}

COPD: chronic obstructive pulmonary disease; NS: nonsmoker; sRAGE: soluble receptor for advanced glycation end-products; AUC: area under the curve; SP-A: surfactant protein A; MPO: myeloperoxidase; NGAL: neutrophil gelatinase-associated lipocalin.

Airway inflammation is important in the development and progression of COPD, with a vast body of evidence supporting the central role of neutrophils in the pathophysiology of this smoking-related syndrome [24]. Myeloperoxidase is a peroxidase enzyme abundantly expressed in the primary granules of neutrophils. Sputum MPO level has been shown to be elevated in patients with COPD and associated with exacerbation of COPD, evidence of a local activation of neutrophils [8, 25, 26]. Sputum levels of MPO were independently correlated with sorputum neutrophils, which is in good agreement with this. Therefore, the present results of sputum MPO could indicate that both overlap and COPD are associated with enhanced airway neutrophilic inflammation.

TABLE 3 Correlations between the plasma and sputum markers and demographics, lung function and sputum cell profile

\begin{tabular}{|c|c|c|c|c|}
\hline & Plasma sRAGE & Plasma SP-A & Sputum MPO & Sputum NGAL \\
\hline Age & -0.046 & $0.420 * *$ & $0.316^{* *}$ & $0.379 * *$ \\
\hline BMI & -0.064 & -0.146 & -0.001 & 0.049 \\
\hline Pack-years & $-0.260 * *$ & $0.541^{* *}$ & $0.276^{*}$ & $0.319 * *$ \\
\hline FEV $1 \%$ pred & $0.262^{* *}$ & $-0.428 * *$ & $-0.291 *$ & $-0.440 * *$ \\
\hline FEV $1 /$ FVC $\%$ & 0.117 & $-0.476^{* *}$ & $-0.232 *$ & $-0.341 * *$ \\
\hline DLco \% pred & $0.447^{* *}$ & $-0.315^{*}$ & $-0.315^{*}$ & $-0.368 * *$ \\
\hline Sputum neutrophils \% & $-0.302^{* *}$ & $0.212^{*}$ & $0.394^{* *}$ & $0.434 * *$ \\
\hline Sputum eosinophils \% & 0.010 & $0.344^{* *}$ & 0.126 & 0.166 \\
\hline
\end{tabular}

SRAGE: soluble receptor for advanced glycation end-products; SP-A: surfactant protein A; MPO: myeloperoxidase; NGAL: neutrophil gelatinase-associated lipocalin; BMI: body mass index; FEV1: forced expiratory volume in $1 \mathrm{~s} ; \%$ pred: \% predicted; FVC: forced vital capacity; DLCO: diffusing capacity of the lung for carbon monoxide. *: $p<0.05 ;{ }^{* *}: \mathrm{p}<0.005$. 
TABLE 4 Multivariate stepwise analysis of all subjects with each plasma or sputum biomarker as the dependent variable

\begin{tabular}{|c|c|c|c|}
\hline & $\boldsymbol{\beta}$ & t-test & p-value \\
\hline \multicolumn{4}{|l|}{ Plasma sRAGE } \\
\hline Dıco \% pred & 0.353 & 2.615 & 0.012 \\
\hline \multicolumn{4}{|l|}{ Plasma SP-A } \\
\hline $\mathrm{FEV}_{1} / \mathrm{FVC} \%$ & -0.370 & -2.796 & 0.008 \\
\hline \multicolumn{4}{|l|}{ Sputum MPO } \\
\hline Neutrophil in sputum \% & 0.371 & 2.908 & 0.005 \\
\hline \multicolumn{4}{|l|}{ Sputum NGAL } \\
\hline FEV1 \% pred & -0.427 & -3.497 & $<0.001$ \\
\hline \multicolumn{4}{|c|}{$\begin{array}{l}\text { sRAGE: soluble receptor for advanced glycation end-products; DLCO: diffusing capacity of the lung for carbon } \\
\text { monoxide; \% pred: \% predicted; SP-A: surfactant protein A; FEV1: forced expiratory volume in } 1 \mathrm{~s} ; \mathrm{FVC} \text { : forced } \\
\text { vital capacity; MPO: myeloperoxidase; NGAL: neutrophil gelatinase-associated lipocalin. \#: multivariate } \\
\text { analysis was adjusted for age, body mass index, current smoking status, pack-years, inhaled corticosteroid } \\
\text { use, and all the significant variables in the univariate analysis. }\end{array}$} \\
\hline
\end{tabular}

The most striking finding of the present study was related to sputum NGAL. Its levels were significantly increased in patients with COPD-asthma overlap compared with COPD. NGAL was originally recognised as an innate immunity antibacterial factor released from neutrophils, but it is also produced by tubular cells in the kidneys and epithelial cells in the intestinal and respiratory tracts [27, 28]. Urinary NGAL is now widely considered to be a sensitive marker for acute kidney injury, as it reflects tubular cell injury [28]. An early study by KEATINGS and BARNES [8] reported elevated sputum NGAL levels in patients with COPD. Bronchial lavage and plasma NGAL levels were also shown to be elevated in patients with COPD [29, 30], and bronchoalveolar lavage fluid NGAL has been associated with emphysema in smokers [31]. NGAL has been demonstrated to exert two functions of potential importance in the pathogenesis of COPD: inhibition of bacterial growth and enhancement of matrix degradation [32]. Thus NGAL could well be associated with COPD, as reflected by the independent correlation of sputum NGAL with airflow obstruction observed in our study. It should be noted that increased sputum NGAL might not be exclusively attributed to activated neutrophils, as the respiratory epithelial cells may also secrete NGAL in response to inflammatory stimuli [27, 33]. Therefore, the sputum NGAL level might be related to neutrophilic inflammation and/or ongoing damage to the respiratory epithelium.

Plasma levels of sRAGE were similarly decreased in the overlap and COPD groups, but not in asthma. In the multivariate analysis, plasma sRAGE levels were independently associated with DLCO. This association is in full agreement with a previous report that reduced plasma sRAGE levels were related to the severity of emphysema [14]. RAGE is primarily expressed on alveolar type 1 pneumocytes and could assist these cells in acquiring a spreading morphology $[34,35]$. Therefore, RAGE might have a role in alveolar integrity, and decreased sRAGE levels could be associated with disrupted alveolar structures. Another possible explanation is that reduction in sRAGE might modify neutrophilic airway inflammation, as sRAGE has antiinflammatory properties by capturing RAGE ligands [36]. This concept is in agreement with the fact that there were reduced plasma sRAGE levels in overlap and COPD with neutrophilic airway inflammation. Moreover, the recent study by SUKKAR et al. [15] has demonstrated a clear association between bronchoalveolar lavage fluid sRAGE and airway neutrophilic inflammation. Therefore, the present results indicate that peripheral lung destruction might be associated with decreased plasma sRAGE in patients with COPD-asthma overlap and COPD, but further investigations are needed to clarify its mechanisms and potential role of modulating the lung inflammation.

A glycoprotein SP-A, synthesised in alveolar type II cells and nonciliated bronchiolar cells of the distal pulmonary epithelium, is secreted into respiratory tract, so the increased circulatory SP-A can be caused by leakage of the lung epithelial barrier [37]. We have previously reported that plasma SP-A concentration is associated with cigarette smoking-related lung injury in smokers [10] and is elevated in patients with COPD [9], the results being in line with previous reports [37-39]. In the current study, both the COPD and overlap groups presented with increased levels of plasma SP-A when compared with patients with asthma and control groups. In agreement with our earlier findings [10], plasma SP-A levels were found to associate with smoking history and the degrees of airway obstruction and peripheral tissue damage. These results suggest that both COPD and COPD-asthma overlap might be associated with smoking-induced lung injury, with the mechanisms being different from those postulated for the pathophysiology of asthma [2]. 
Recently, we and others have reported that COPD-asthma overlap is associated with significantly lower health-related quality of life and increased exacerbation rates compared with COPD, which could not be explained by patients' demographic, lung function or computed tomography findings of emphysema $[5,6]$. In the present study, plasma SP-A and sputum MPO levels were elevated, while plasma sRAGE was decreased in both COPD-asthma overlap and COPD when compared with asthma. Moreover, only sputum NGAL was significantly elevated in overlap patients compared with all of the other groups including COPD. Taken together, these results of the COPD-related biomarkers suggest that while airway neutrophilic inflammation and peripheral lung destruction are common pathological processes in overlap and COPD, COPD-asthma overlap is associated with enhanced airway inflammation, which can be detected by increased levels of sputum NGAL. These findings might have implications for the distinct clinical characteristics of COPD-asthma overlap, but further investigation will be needed to clarify the mechanisms of airway inflammation and the optimal monitoring and treatment strategy of overlap patients.

This was a cross-sectional study and, therefore, further prospective investigations would be required to evaluate the association of these plasma and sputum markers with the development of chronic airway diseases. Basic research studies are warranted to explore mechanisms behind this association. This study has significant strengths. First, none of the control subjects had any other exposures. Secondly, the diagnosis of asthma, COPD and COPD-asthma overlap was based on both clinical history and strict and objective criteria, which enables unbiased comparisons of the characteristics between those diseases. Although the sample size was limited, the present results do seem to reveal the difference in the biomarkers of COPDasthma overlap compared with asthma and COPD alone.

In conclusion, we have demonstrated that patients with COPD-asthma overlap and COPD have a similar biomarker profile in plasma SP-A and sRAGE and sputum MPO levels, which differ from that in patients with asthma. Moreover, elevated levels of sputum NGAL might be a characteristic feature of overlap that could differentiate overlap from COPD, suggesting enhanced neutrophilic airway inflammation and/or airway epithelial injury in COPD-asthma overlap.

\section{Acknowledgements}

T. Marjomaa and S. Nummijoki (Dept of Medicine, Pulmonary Division, University of Helsinki and Helsinki University, Central Hospital, Helsinki, Finland) are acknowledged for their help and/or excellent technical assistance. We thank P. Piirilä (Clinical Physiology Laboratory, Dept of Clinical Physiology and Nuclear Medicine, Helsinki University Central Hospital, Helsinki, Finland) for her help in accessing lung function tests.

\section{References}

1 Rabe KF, Hurd S, Anzueto A, et al. Global strategy for the diagnosis, management, and prevention of chronic obstructive pulmonary disease: GOLD executive summary. Am J Respir Crit Care Med 2007; 176: 532-555.

2 Bateman ED, Hurd SS, Barnes PJ, et al. Global strategy for asthma management and prevention: GINA executive summary. Eur Respir J 2008; 31: 143-178.

3 Gibson PG, Simpson JL. The overlap syndrome of asthma and COPD: what are its features and how important is it? Thorax 2009; 64: 728-735.

4 Soriano JB, Davis KJ, Coleman B, et al. The proportional Venn diagram of obstructive lung disease: two approximations from the United States and the United Kingdom. Chest 2003; 124: 474-481.

5 Kauppi P, Kupiainen H, Lindqvist A, et al. Overlap syndrome of asthma and COPD predicts low quality of life. J Asthma 2011; 48: 279-285.

6 Hardin M, Silverman EK, Barr RG, et al. The clinical features of the overlap between COPD and asthma. Respir Res 2011; 12: 127.

Rosenberg SR, Kalhan R. Biomarkers in chronic obstructive pulmonary disease. Transl Res 2012; 159: 228-237.

Keatings VM, Barnes PJ. Granulocyte activation markers in induced sputum: comparison between chronic obstructive pulmonary disease, asthma, and normal subjects. Am J Respir Crit Care Med 1997; 155: 449-453.

9 Ilumets H, Mazur W, Toljamo T, et al. Ageing and smoking contribute to plasma surfactant proteins and protease imbalance with correlations to airway obstruction. BMC Pulm Med 2011; 11: 19.

10 Mazur W, Toljamo T, Ohlmeier S, et al. Elevation of surfactant protein A in plasma and sputum in cigarette smokers. Eur Respir J 2011; 38: 277-284.

11 Ohlmeier S, Vuolanto M, Toljamo T, et al. Proteomics of human lung tissue identifies surfactant protein A as a marker of chronic obstructive pulmonary disease. J Proteome Res 2008; 7: 5125-5132.

12 Ohlmeier S, Mazur W, Salmenkivi K, et al. Proteomic studies on receptor for advanced glycation end product variants in idiopathic pulmonary fibrosis and chronic obstructive pulmonary disease. Proteomics Clin Appl 2010; 4: 97-105.

13 Smith DJ, Yerkovich ST, Towers MA, et al. Reduced soluble receptor for advanced glycation end-products in COPD. Eur Respir J 2011; 37: 516-522.

14 Miniati M, Monti S, Basta G, et al. Soluble receptor for advanced glycation end products in COPD: relationship with emphysema and chronic cor pulmonale: a case-control study. Respir Res 2011; 12: 37.

15 Sukkar MB, Wood LG, Tooze M, et al. Soluble RAGE is deficient in neutrophilic asthma and COPD. Eur Respir J 2012; 39: 721-729.

16 Laitinen T, Hodgson U, Kupiainen $\mathrm{H}$, et al. Real-world clinical data identifies gender-related profiles in chronic obstructive pulmonary disease. COPD 2009; 6: 256-262. 
Enright P, Skloot G, Herbert R. Standardization of spirometry in assessment of responders following man-made disasters: World Trade Center worker and volunteer medical screening program. Mt Sinai J Med 2008; 75: 109-114.

18 Viljanen AA, Halttunen PK, Kreus KE, et al. Spirometric studies in non-smoking, healthy adults. Scand J Clin Lab Invest Suppl 1982; 159: 5-20.

19 British Thoracic Society Scottish Intercollegiate Guidelines Network. British Guideline on the Management of Asthma. Thorax 2008; 63: Suppl. 4, iv1-iv121.

20 Miller MR, Hankinson J, Brusasco V, et al. Standardisation of spirometry. Eur Respir J 2005; 26: 319-338.

21 Djukanovic R, Sterk PJ, Fahy JV, et al. Standardised methodology of sputum induction and processing. Eur Respir J 2002; 20: Suppl. 37, 1s-2s.

22 Kelly MM, Keatings V, Leigh R, et al. Analysis of fluid-phase mediators. Eur Respir J 2002; 20: Suppl. 37, 24s-39s.

23 Louhelainen N, Stark H, Mazur W, et al. Elevation of sputum matrix metalloproteinase-9 persists up to 6 months after smoking cessation: a research study. BMC Pulm Med 2010; 10: 13

24 Barnes PJ. Immunology of asthma and chronic obstructive pulmonary disease. Nat Rev Immunol 2008; 8: 183-192.

25 Aaron SD, Angel JB, Lunau M, et al. Granulocyte inflammatory markers and airway infection during acute exacerbation of chronic obstructive pulmonary disease. Am J Respir Crit Care Med 2001; 163: 349-355.

26 Liesker JJ, Bathoorn E, Postma DS, et al. Sputum inflammation predicts exacerbations after cessation of inhaled corticosteroids in COPD. Respir Med 2011; 105: 1853-1860.

27 Cowland JB, Sorensen OE, Sehested M, et al. Neutrophil gelatinase-associated lipocalin is up-regulated in human epithelial cells by IL-1 beta, but not by TNF-alpha. J Immunol 2003; 171: 6630-6639.

28 Bolignano D, Donato V, Coppolino G, et al. Neutrophil gelatinase-associated lipocalin (NGAL) as a marker of kidney damage. Am J Kidney Dis 2008; 52: 595-605.

29 Eagan TM, Damas JK, Ueland T, et al. Neutrophil gelatinase-associated lipocalin: a biomarker in COPD. Chest 2010; 138: 888-895.

30 Ekberg-Jansson A, Andersson B, Bake B, et al. Neutrophil-associated activation markers in healthy smokers relates to a fall in DL(CO) and to emphysematous changes on high resolution CT. Respir Med 2001; 95: 363-373.

31 Betsuyaku T, Nishimura M, Takeyabu K, et al. Neutrophil granule proteins in bronchoalveolar lavage fluid from subjects with subclinical emphysema. Am J Respir Crit Care Med 1999; 159: 1985-1991.

32 Yan L, Borregaard N, Kjeldsen L, et al. The high molecular weight urinary matrix metalloproteinase (MMP) activity is a complex of gelatinase B/MMP-9 and neutrophil gelatinase-associated lipocalin (NGAL). Modulation of MMP-9 activity by NGAL. J Biol Chem 2001; 276: 37258-37265.

33 Karlsen JR, Borregaard N, Cowland JB. Induction of neutrophil gelatinase-associated lipocalin expression by costimulation with interleukin-17 and tumor necrosis factor- $\alpha$ is controlled by $\mathrm{I} \kappa \mathrm{B}-\zeta$ but neither by $\mathrm{C} / \mathrm{EBP}-\beta$ nor $\mathrm{C} /$ EBP- $\delta$. J Biol Chem 2010; 285: 14088-14100.

34 Dahlin K, Mager EM, Allen L, et al. Identification of genes differentially expressed in rat alveolar type I cells. Am J Respir Cell Mol Biol 2004; 31: 309-316.

35 Demling N, Ehrhardt C, Kasper M, et al. Promotion of cell adherence and spreading: a novel function of RAGE, the highly selective differentiation marker of human alveolar epithelial type I cells. Cell Tiss Res 2006; 323: 475-488.

36 Zhang H, Tasaka S, Shiraishi Y, et al. Role of soluble receptor for advanced glycation end products on endotoxininduced lung injury. Am J Respir Crit Care Med 2008; 178: 356-362.

37 Robin M, Dong P, Hermans C, et al. Serum levels of CC16, SP-A and SP-B reflect tobacco-smoke exposure in asymptomatic subjects. Eur Respir J 2002; 20: 1152-1161.

38 Kida K, Oda H, Yamano Y, et al. Effects of cigarette smoking on the serum concentration of lung surfactant protein A (SP-A). Eur Respir J 1997; 10: 2124-2126.

39 Kobayashi H, Kanoh S, Motoyoshi K. Serum surfactant protein-A, but not surfactant protein-D or KL-6, can predict preclinical lung damage induced by smoking. Biomarkers 2008; 13: 385-392. 